\title{
Low-Loss Quantum Buses for Photons
}

\section{Researchers demonstrate a way to shuttle photons between modules of a quantum processor that can detect and correct communication losses.}

\author{
By Rachel Berkowitz
}

F uture quantum computers will contain many qubits that will all need to interact with one another in a controlled way while remaining isolated. One approach toward realizing such a system is to build it in a modular fashion, meaning that parts of the computer are housed in separate containers, or modules. The key challenge in building a modular quantum computer is developing robust devices to transfer information between modules. Luke Burkhart at Yale University and colleagues now present a low-loss prototype hardware and method for shuttling qubits between modules via a coaxial cable [1].

Burkhart and his colleagues started with two microwave cavities, which each hosted a superconducting qubit. They then linked the cavity modules with a coaxial cable. The cable served as a resonance cavity that acted as a quantum "bus," shuttling information between components.

To demonstrate their approach, the team applied microwave pulses to each qubit, switching on interactions between the

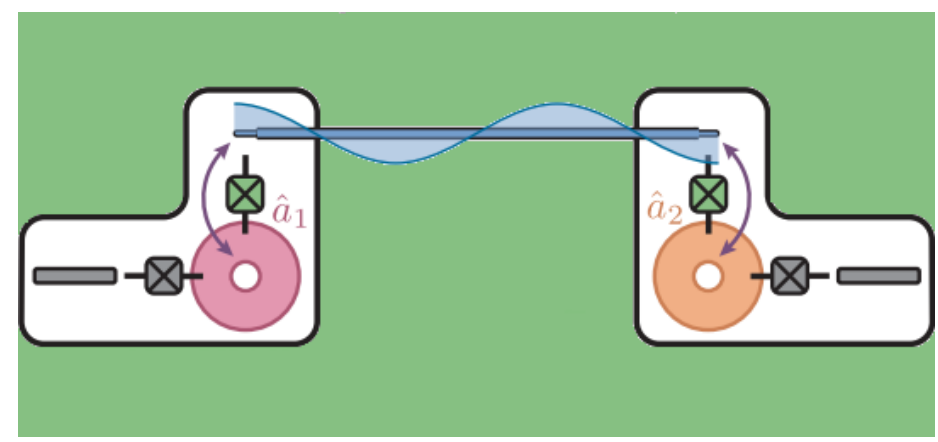

Credit: L. D. Burkhart et al. [1] modules and the cable that controlled the flow of information between them. They then performed error correction on the transferred data, measuring the parity of the number of photons-whether the number is even or odd-to determine whether loss errors had occurred.

The team showed that their error-corrected information-transfer protocol performs on par with uncorrectable methods. They say that the design provides a pathway toward robust operations, which will allow quantum computers to operate with fewer qubits.

Rachel Berkowitz is a Corresponding Editor for Physics based in Vancouver, Canada.

\section{REFERENCES}

1. L. D. Burkhart et al., "Error-detected state transfer and entanglement in a superconducting quantum network," PRX Quantum 2, 030321 (2021). 